\title{
$1 \quad$ PhyB induces intron retention and uORF-mediated translational inhibition of PIF3
}

2

3 Jie Dong ${ }^{1}$, Haodong $\mathrm{Chen}^{2}$, Xing Wang Deng ${ }^{2}$, Vivian F. $\operatorname{Irish}^{1}$, Ning Wei ${ }^{1, *}$

$4{ }^{1}$ Department of Molecular, Cellular and Developmental Biology, Yale University, New

$5 \quad$ Haven, Connecticut 06520, USA

$6 \quad{ }^{2}$ State Key Laboratory of Protein and Plant Gene Research, Peking-Tsinghua Center for Life

7 Sciences, School of Advanced Agricultural Sciences and School of Life Sciences, Peking

8 University, Beijing 100871, China

$9 \quad *$ Address Correspondence to ning.wei@yale.edu (Ning Wei)

10

\section{Author Contributions}

12 J.D. designed and performed the experiments with suggestions from N.W.; V.I. and X.W.D

13 provided the resources; J.D. and N.W. wrote the manuscript. V.I., X.W.D., J.D., H.C., and

14 N.W. edited the final manuscript. Ning Wei agrees to serve as the author for contact and ensures communication.

Running Title: phyB inhibits PIF3 production via AS coupled uORF

17

One Sentence Summary: Light down-regulates PIF3 by multiple mechanisms. We show that phyB induces an alternative splicing event that inhibits PIF3 protein translation, and that is regulated by short-day diurnal cycle.

21

22 Key words: phytochrome B, PIF3, translational regulation, alternative splicing, intron 23 retention, uORF, photomorphogenesis, short day, diurnal cycle, light regulation, 24 Arabidopsis. 
Abstract

The phytochrome B (phyB) photoreceptor stimulates light responses in plants in part by inactivating repressors of light responses such as phytochrome-interacting factor 3 (PIF3). It has been established that activated phyB inhibits PIF3 by rapid protein degradation and decreased transcription. PIF3 protein degradation has been shown to be mediated by EIN3-BINDING F-BOX PROTEIN (EBF) and LIGHT-RESPONSE BTB (LRB) E3 ligases, the latter simultaneously targeting phyB for degradation. In this study, we show that PIF3 level is additionally regulated by alternative splicing and protein translation. Overaccumulation of photo-activated phyB, which occur in the mutant defective for $L R B$ genes under continuous red light (Rc), induces a specific alternative splicing of PIF3 that results in retention of an intron in the 5'UTR of PIF3 mRNA. In turn, the upstream opening reading frames (uORF) contained within this intron inhibit PIF3 protein synthesis. The phyB-dependent alternative splicing of PIF3 is diurnally regulated under the short-day light cycle. We hypothesize that this reversible regulatory mechanism may be utilized to fine-tune the level of PIF3 protein in light-grown plants, and may contribute to the oscillation of PIF3 protein abundance under the short-day environment.

\section{Introduction}

The phytochromes (phy) are important photoreceptors regulating plant growth and development throughout its lifecycle. Among the five members of the phytochrome family in Arabidopsis, phyB is relatively abundant in light and plays a major role in controlling hypocotyl growth under prolonged continuous red light ( $\mathrm{Rc}$ ) (Li et al., 2011). Phytochrome-Interacting Factors (PIFs) are basic helix-loop-helix transcription factors that function to repress light responses (Leivar et al., 2008; Shin et al., 2009; Pham et al 2018). PIF3, the first characterized member of the PIF family (Ni et al., 1998), accumulates abundantly in dark-grown seedlings to maintain etiolation, and it rapidly declines upon light exposure, allowing establishment of photomorphogenesis in seedlings (Bauer et al., 2004;

53 Zhang et al., 2013). During de-etiolation, photoactivated phyB directly binds to PIF3 and

54 induces PIF3 phosphorylation, which are necessary for the $26 \mathrm{~S}$ proteasome-mediated 
degradation of PIF3 (Al-Sady et al., 2006; Ni et al., 2013). In addition, phyB down-regulates PIF3 transcription by about 3-fold during de-etiolation and interferes with PIF3 DNA binding ability (Shi et al., 2016; Park et al., 2018). Inhibition of PIF3 at the level of translation has not been reported, although light signals generally promote translation activity globally (Paik et al., 2012; Chen et al., 2018).

After seedling establishment, normal light-grown plants maintain steady-state lower expression of PIFs, which continue to modulate plant development in response to light and temperature (Leivar and Monte, 2014; Pham et al., 2018). For examples, PIF3 was shown to play prominent roles in promoting dark-period elongation growth under short-day conditions (Soy et al., 2012; 2016), and in freezing tolerance (Jiang et al., 2017).

Studies on phyB induced rapid protein degradation of PIF3 have revealed that PIF3 can be degraded through at least two pathways. During de-etiolation, F-box proteins EIN3-BINDING F BOX PROTEIN (EBF) mediate PIF3 degradation via $\mathrm{SCF}^{\mathrm{EBFs}}$ ubiquitin E3 ligases to facilitate photomorphogenesis of plants (Dong et al., 2017). Plants deficient for EBFs exhibit reduced light sensitivity and inefficient de-etiolation. Under high fluence red light, PIF3 can also be degraded via LIGHT-RESPONSE BTBs (LRBs), the ubiquitin E3 ligases (CRL3 ${ }^{\mathrm{LRBs}}$ ) that target phyB for degradation to attenuate light responses (Ni et al., 2014). Plants deficient for LRBs show hypersensitivity to light in a phyB dependent manner (Christians et al., 2012; Ni et al., 2014; Dong et al., 2017).

To study the functional dynamics between EBFs and LRBs in the regulation of PIF3, we eliminated both types of PIF3 E3 ligases and generated a ein3 ebf lrb mutant line. Using this mutant line, we uncovered an unexpected mechanism by which phyB inactivates PIF3: i.e. inhibiting PIF3 protein translation via alternative splicing (AS).

\section{Results and Discussion}

80 To knock-out both ubiquitin E3 families of PIF3, we generated ein3 ebf lrb hextuple mutant 81 by crossing the viable ein3 ebfl ebf2 (ein3 ebf) mutant with lrb1 lrb2 lrb3 (lrb) (Figure 1). 
(Supplemental Figure 1). The resulting phenotypes under continuous red light (Rc) showed that the ein3 ebf mutant exhibited longer hypocotyls compared to ein3, while lrb showed shorter hypocotyls compared to Col (Figure 1A and 1B), consistent with previous observations (Dong et al., 2017; Ni et al., 2014; Christians et al., 2012). The ein3 ebf lrb mutant almost phenocopied $l r b$ in having a shortened hypocotyl under Rc, suggesting that lrb strongly suppressed ein3 ebf (Figure 1A and 1B). The hyper-photosensitive phenotype of ein3 ebf lrb seemed at odds with the anticipated accumulation of PIF3 in high levels, and prompted us to examine the PIF3 protein levels in these mutants.

By eliminating both E3 ubiquitin ligase families that regulate PIF3 abundance, one would expect that PIF3 protein would be stabilized in the ein3 ebf lrb mutant. However, western blot showed that PIF3 protein accumulated only in ein3 ebf, but not in lrb nor in ein3 ebf lrb mutants (Figure 1C and 1D). PIF3 protein levels were much higher in ein3 ebf than in ein3 ebf lrb, while PIF3 transcript levels were comparable between these two mutant lines (Figure 1D and 1E). In addition, PIF3 activities matched with PIF3 protein levels in these mutants, based on the expression of the PIF3 target genes XTR7 and XTH33 (Figure $1 \mathrm{~F}$ and $1 \mathrm{G}$ ). In the dark, relative levels of PIF3 protein corresponded with their respective mRNA levels among the mutants tested (Supplemental Figure 2), suggesting the regulation of PIF3 protein levels by LRBs and EBFs are light-dependent. Taken together, the $l r b$ mutations appeared to have suppressed the PIF3 over-accumulation of ein3 ebf by decreasing PIF3 protein expression without affecting its transcript levels, and one possibility is that $l r b$ could potentially inhibit translation of PIF3 under Rc. As lrb mutations primarily cause phyB accumulation and light hypersensitivity (Christians et al., 2012; Ni et al., 2014), we hypothesized that phyB may negatively regulate PIF3 translation.

Light causes wide-spread alternative splicing (AS) in plant transcriptome (Shikata et al., 2014; Hartmann et al., 2016; Xin et al., 2017; Godoy Herz et al., 2019). Although PIF3 has not been experimentally demonstrated to undergo light regulated AS, based on the information from Araport (Arabidopsis Information Portal: www.araport.org), transcripts with alternative splicing patterns at an intron of approximately 500 bp in PIF3 5, 
111 untranslated region (UTR) have been found in the database (Figure 2A). We refer to this

112 intron as Intron ${ }_{\mathrm{AS}}$ in this study, and monitored its splicing level by RT-qPCR. We found that

113 the Intron ${ }_{\mathrm{AS}}$-retained form was significantly increased relative to total PIF3 mRNA in Col

114 under Rc, as compared to dark (Dc; Figure 2B), suggesting that Intron $_{\mathrm{AS}}$ retention in PIF3

115 mRNA is a light-dependent event. Furthermore, mutation of phyB essentially abolished the

116 light $(\mathrm{Rc})$ induced increase of Intron $_{\mathrm{AS}}$ retention (Figure $2 \mathrm{~B}$ ), indicating that phyB is critical

117 in red light responsive Intron $_{\mathrm{AS}}$ retention in PIF3 mRNA. Moreover, $l r b$ mutations caused

118 higher Intron ${ }_{\mathrm{AS}}$ retention under Rc (Supplemental Figure 3), which is consistent with higher

119 levels of phyB accumulation in lrb (Christians et al., 2012; Ni et al., 2014).

120 To better visualize the retention of Intron $_{\mathrm{AS}}$, we performed gel electrophoresis of the

121 PCR products using a primer pair that would produce a longer product when Intron $_{\mathrm{AS}}$ is

122 retained, and a shorter product when Intron $_{\mathrm{AS}}$ is spliced (Figure 2C). The ACTIN2 (ACT2)

123 primer pair produced only the short-sized band from all cDNA samples while produced a

124 long-sized band from genomic DNA (Figure 2C), suggesting there was no intron retention

125 in ACT2 mRNA in those samples. By contrast, the PIF3 primer sets produced both the

126 intron-containing and intron-spliced bands from the cDNA samples. The Intron $_{\mathrm{AS}}$-retained

127 form was strongly enriched under Rc compared to Dc in Col, but the light-induced intron

128 retention was nearly absent in phyB-9. This result shows again that Intron $_{\mathrm{AS}}$ retention in

129 PIF3 mRNA occurred under red light, and that this event was dependent on phyB (Figure

130 2C). As an additional control, we examined other introns in PIF3 mRNA and found no

131 evidence of retention of any of other introns (Supplemental Figure 4). These data show that

132 photoactivated phyB can induce an alternative splicing event that results in the retention of

133 Intron $_{\mathrm{AS}}$ in PIF3 mRNA.

134 Extensive studies have shown that the 5'UTR can influence its mRNA translation

135 (Araujo et al., 2012; von Arnim et al., 2014). Since Intron $_{\mathrm{AS}}$ resides within the 5'UTR of

136 PIF3 mRNA, we next asked whether the retention of Intron $_{\mathrm{AS}}$ may affect PIF3 translation.

137 We generated T7 promoter-driven constructs in which either normally spliced PIF3 5' UTR

138 (5U) or Intron $_{\mathrm{AS}}$-retained 5'UTR (5IU) was inserted upstream of the PIF3 coding sequence. 
139 In a mammalian cell-based in vitro translation assay using either DNA or RNA as template,

140 5U produced higher levels of PIF3 proteins than did 5IU (Figure 3A). Clearly, retaining the

141 Intron $_{\mathrm{AS}}$ sequence in PIF3 5'UTR inhibited protein translation even in a heterologous in

142 vitro translation system.

143 To test the effect of Intron $_{\mathrm{AS}}$ in planta, we generated transgenic plants in a pif3-3

144 mutant background that carried the following transcription cassettes under the 35S promoter:

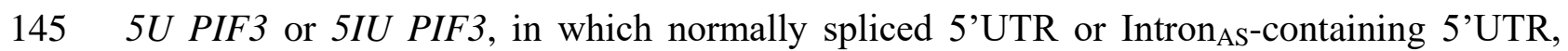

146 respectively, were placed upstream of PIF3 coding region (Figure 3B). Three independent

$1475 U$ PIF3 and 5IU PIF3 transgenic lines with similar levels of total PIF3 mRNA transcript

148 were selected, and Intron ${ }_{\mathrm{AS}}$-containing PIF3 transcript was confirmed to exist only in $5 I U$

149 PIF3 transgenic lines (Supplemental Figure 5). It appeared that, in the context of this

150 artificial Intron ${ }_{\mathrm{AS}}$-containing PIF3 transgene, Intron $_{\mathrm{AS}}$ could be spliced but very

151 inefficiently, as most of the transcript retained the intron (Supplemental Figure 5C). In

152 darkness when PIF3 protein was stable, western blot showed that PIF3 protein expressed in

153 5U PIF3 lines, but was almost undetectable in 5IU PIF3 lines (Figure 3B). Quantification

154 showed that retention of Intron $_{\mathrm{AS}}$ in PIF3 5'UTR caused an approximately $80 \%$ reduction in

155 PIF3 protein levels (Figure 3B). These results indicate that forced retention of Intron $_{\mathrm{AS}}$

156 upstream of the PIF3 coding sequence inhibits the protein production. As a result, the 5IU

157 PIF3 transgene was unable to restore the phenotype of pif3-3 in hypocotyl elongation under

158 Rc, in contrast to the rescue seen with 5U PIF3 (Figure 3C and 3D). We deduce from these

159 results that the retention of Intron $_{\mathrm{AS}}$ in the 5'UTR of PIF3 mRNA can inhibit PIF3 protein

160 expression both in vitro and in vivo, consequently affecting PIF3-regulated hypocotyl

161 elongation.

162 Upstream Open Reading Frame (uORF)-mediated translation inhibition has been shown

163 to regulate gene expression involved in light response as well as other developmental and

164 metabolic processes in plants (von Arnim et al., 2014; Kurihara et al., 2018). The PIF3

165 Intron $_{\mathrm{AS}}$ sequence contains multiple AUG start codons and putative uORFs (Supplemental

166 Figure 6). The longest uORF, which is 129 nucleotides in length just upstream of the PIF3 
167 main ORF, is considered the most likely candidate uORF to inhibit downstream PIF3

168 translation, even though the start codon context is suboptimal (Kozak, 2001). To test this

169 idea, we carried out a dual-luciferase protoplast transient assay where the 5'UTR testing

170 effector was inserted upstream of firefly luciferase (LUC) reporter, with a 35S promoter

171 driven Renilla luciferase (RLUC) as the internal control (Figure 3E). The testing effectors

172 include: PIF3 5'UTR (5U), Intron ${ }_{\mathrm{AS}}$-containing 5'UTR (5IU), or Intron ${ }_{\mathrm{AS}}$ with the mutated

173 form of $\mathrm{uORF}$ start codon $5 \mathrm{I}_{\mathrm{AS}} \mathrm{U}$ (5IUm). In another set, we explicitly tested the effect of

174 the uORF without other Intron ${ }_{\mathrm{AS}}$ sequences: either the uORF with an ATG start codon, or

175 mutated uORF with CTG (uorf). The data showed that relative LUC activity in 5IU and

176 uORF was significantly lower than that in 5IUm and uorf, respectively (Figure 3E). These

177 results indicate that the $129 \mathrm{nt} \mathrm{uORF}$ of Intron $_{\mathrm{AS}}$ can inhibit the translation of the

178 downstream ORF.

179 We noticed that the inhibitory effect of Intron $_{\mathrm{AS}}$-inclusion was not as strong in this 180 transient assay (Fig. 3E 5U vs 5IU) compared to the in planta assay (Fig. 3B), which was

181 probably due to the difference in the assay system. In addition, the dramatic inhibition of

182 protein expression seen in the experiments of Figure 3A and $3 \mathrm{~B}$ could be caused by the 183 combination of the uORFs in Intron $_{\mathrm{AS}}$, as oppose to Figure 3E that tested the $129 \mathrm{nt} \mathrm{uORF}$ 184 exclusively. Regardless, our data support the conclusion that retention of Intron $_{\mathrm{AS}}$, which 185 contains uORFs, causes a blockage in PIF3 expression at the level of translation.

186 It is well established that phyB activation leads to a sharp decline in PIF3 abundance 187 during de-etiolation (Bauer et al., 2004; Al-Sady et al., 2006; Dong et al., 2017). phyB

188 stimulated PIF3 protein degradation accounts for predominant portion of PIF3 189 downregulation (Al-Sady et al., 2006), while decreased transcription of PIF3 also occurs 190 (Shi et al., 2016). Here, our data show that phyB can additionally inhibit PIF3 protein 191 translation via retention of Intron $_{\mathrm{AS}}$ under prolonged red light. However, questions remain 192 as to how important the translation control is relative to the control of protein stability and 193 transcription, and under what natural circumstances would the plants utilize this type of 194 regulatory mechanism. To this end, we examined the kinetics of Intron $_{\mathrm{As}}$ retention rate 
195 during de-etiolation. When dark-grown seedlings were irradiated with red light, PIF3

196 protein level rapidly and sharply declined within minutes and dropped more than 10 fold

197 within hours after light exposure, while the increase of Intron $_{\mathrm{AS}}$ retention was more evident

198 in prolonged light-grown plants than during the 24 hours of de-etiolation (Supplemental

199 Figure 7). This result suggests that phyB-induced AS is unlikely to play a major role in

200 de-etiolation, but maybe a feature associated with light-grown plants. As most

201 light-dependent AS profiling studies were performed under the de-etiolation condition

202 (Shikata et al., 2014; Hartmann etal., 2016; Kurihara et al., 2018), this maybe a reason why

203 PIF3 has not been reported to undergo light-induced AS.

204 In growth plants, PIF3 protein abundance has been shown to oscillate under short day

205 diurnal cycle to promote pre-dawn hypocotyl growth (Soy et al., 2012; 2016). Interestingly,

206 unlike PIF4 and PIF5 whose transcription is strongly diurnally regulated, transcription

207 regulation of PIF3 is minimum under this condition, while PIF3 protein accumulates in the

208 dark period and decline during the day (Soy et al., 2012). We examined PIF3 Intron AS

209 retention in plants grown in short-day light cycle. The data showed that the Intron $_{\mathrm{AS}}$

210 retention rate of PIF3 oscillated diurnally, rising post-dawn and dropping around dusk

211 (Figure 4A). This pattern would correspond with a reduced PIF3 translation during the day,

212 and normal protein synthesis during the night, which is consistent with peak accumulation

213 of PIF3 protein at pre-dawn (Soy et al., 2012). We thus suggest that regulation of PIF3

214 protein synthesis, in coordination with PIF3 degradation, contributes to diurnal oscillation

215 of PIF3 protein levels under short-day conditions.

216 In summary, we have identified a new regulatory mechanism of phyB on PIF3, and

217 revealed that light signals can inactivate PIF3 at level of translation, apart from regulating

218 its transcription, DNA binding, and protein stability (Figure 4B). In particular, our findings

219 explain why the $l r b$ E3 mutations can suppress the ebf E3 mutants phenotypically and at the

220 level of PIF3 protein accumulation. This is because $l r b$ induced phyB accumulation leads to

221 a block of PIF3 protein synthesis, which would alleviate the problems caused by the 
223 cause the UORF inclusion in the 5'UTR of several genes as a result of global change of

224 transcription start sites (Kurihara et al., 2018). In PIF3, alternative transcription initiation

225 may also occur (Figure 2A), but it is not related to the inclusion of the uORFs. Regarding

226 the extent of translational regulation relative to transcription and protein degradation of

227 PIF3, we suggest that, during de-etiolation, the massive irreversible PIF3 protein

228 degradation probably accounts for most of the rapid and dramatic drop of PIF3 levels, while

229 the AS coupled-translational regulation is minor if it occurs at all. In grown plants, PIF3

230 expresses at a reduced level, and a reversible control mechanism as revealed in this study

231 may operate to fine-tune PIF3 levels, likely in conjunction with other PIF3 regulatory

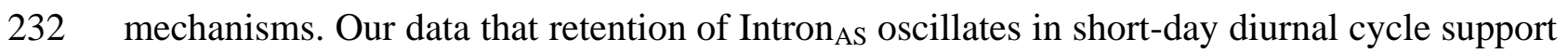

233 this idea. It is possible that Intron $_{\mathrm{AS}}$-coupled translational regulation of PIF3 may play

234 important roles in other light modulated physiological processes in plants.

235

236

237 


\section{Materials and Methods}

\section{Plant materials and growth condition}

240 Arabidopsis thaliana mutants are in the Col (Columbia) ecotype and were grown under long 241 day conditions ( $16 \mathrm{~h}$ day/ $8 \mathrm{~h}$ dark) at $22{ }^{\circ} \mathrm{C}$. The parental lines of the higher order mutant, 242 ein3 ebf1 ebf2 (ein3 ebf) and lrb1 lrb2 lrb3 (lrb), were previously described in An et al., 2432010 and Ni et al., 2014, respectively. The pif3 mutant was described in Dong et al., 2017.

244 Seeds were surface sterilized using 30\% Bleach and sowed on half-strength MS plate 245 (2.2 g/L Murashige and Skoog Basal Salt, $0.5 \mathrm{~g} / \mathrm{L}$ MES, $8 \mathrm{~g} / \mathrm{L}$ agar, pH 5.7). The seeds were 246 then stratified at $4{ }^{\circ} \mathrm{C}$ for 2 days, induced germination for $3 \mathrm{~h}$ under white light, and either 247 put into darkness or $10 \mu \mathrm{mol} / \mathrm{m}^{2} / \mathrm{s}$ continuous red light for 3-4 days followed by phenotypic observation, RT-qPCR analysis, and western blot analysis.

\section{Generation of transgenic plants}

251

For generation of 35S: $5 U$ PIF3 and 35S: 5IU PIF3, 5U PIF3 coding sequence was amplified directly from cDNA, while 5IU PIF3 coding sequence was generated by overlapping PCR of 5IU, which was amplified from genomic DNA, and PIF3 coding sequence, which was amplified from cDNA. The resulting 5U PIF3 and 5IU PIF3 coding sequences were inserted into pjim19 (Kan) using XhoI/SacI restriction enzymes. The vectors were then transformed into the pif3-3 background using Agrobacterium tumefasciens strain GV3101 by the floral dip method. Primers used were listed in Supplemental Table 1.

\section{RT-qPCR}

261 Total RNA was extracted using Qiagen RNeasy Plant Mini kit according to the instructions.

262 Reverse transcription was performed using SuperScript III reverse transcriptase (Invitrogen), 263 oligo(dT) 12-18 (Invitrogen) and $1 \mu \mathrm{g}$ total RNA as template. The resulting cDNA samples 264 were diluted 10-fold with nuclease-free water, and $2 \mu 1$ was used for qPCR assay in CFX96 real-time system (Bio-rad). ACT2 was used as internal control. Primers used for RT-qPCR 
were listed in Supplemental Table 1.

\section{Immunoblot}

269 Total protein was extracted from whole seedlings using denaturing buffer ( $8 \mathrm{M}$ urea, $0.1 \mathrm{M}$

$270 \mathrm{NaH}_{2} \mathrm{PO}_{4}, 0.1 \mathrm{M}$ Tris- $\mathrm{HCl}, \mathrm{pH} 8.0$ ). Protein concentration was determined by the Bio-rad

271 protein assay. The same amount of total protein was subjected to SDS-PAGE, transferred to PVDF film, blocked by 5\% milk in 1×TBST, and incubated with anti-PIF3 or anti-RPN6 at $4^{\circ} \mathrm{C}$ overnight. After wash, film was further incubated with HRP-conjugated secondary antibody for $1 \mathrm{~h}$ at RT and exposed to X-ray film. The X-ray film was then developed and fixed in a dark room.

\section{In vitro translation (IVT) assay}

278 For DNA as template, the 5U PIF3 and 5I1U PIF3 coding sequence was inserted into pT7CFE1-Myc (Pierce) vector using NdeI/SacI, yielding pT7-5U PIF3 and pT7-5I1U PIF3, respectively. $1 \mu \mathrm{g}$ DNA was used as template in a $25 \mu \mathrm{l}$ in vitro translation system following the instructions of 1-step human coupled IVT kit-DNA (Pierce). The resulting IVT product was mixed with $100 \mu$ l loading buffer, boiled, and subjected to western blot analysis using anti-PIF3 antibody. Ponceau S staining was used as loading control. Primers used for construct cloning were listed in Supplemental Table 1.

For RNA as template, the pT7-5U PIF3 and pT7-5I1U PIF3 plasmids were linearized by

Not I digestion and purified using QIAquick PCR purification kit. $1 \mu \mathrm{g}$ linearized plasmid was used as template for in vitro transcription using HiScribe ${ }^{\mathrm{TM}} \mathrm{T} 7$ High Yield RNA Synthesis Kit (NEB). $5 \mu \mathrm{g}$ RNA was used as template for IVT, and western blot was done the same as DNA as template.

\section{Dual luciferase assay}

292 The 35S promoter was cloned into pGreenii 0800-LUC using Hind III/BamH I, yielding 
294 (5IUm), uORF and mutated uORF (uorf) of PIF3 were cloned into pGreenii 35S-LUC using

295 BamH I/ Spe I. Primers used were listed in Supplemental Table 1.

296 Protoplasts were isolated based on a protocol previously described (Yoo et al., 2007).

297 The leaves of Col adult plants grown under LD condition for 3-4 weeks were used for

298 protoplast isolation. $10 \mu \mathrm{g}$ plasmids were used for transfection. After incubated in the dark

299 for $12 \mathrm{~h}$, luciferase activity was measured following the instructions of Dual-Luciferase ${ }^{\circledR}$

300 Reporter Assay System (Promega). Each plasmid was measured with 4 biological replicates

301 and at least 2 technical repeats.

302

303 Acknowledgements

304 We thank Prof. Peter Quail and Dr. Weimin Ni for providing lrb1 lrb2 lrb3 mutant seeds.

305 We also thank Prof. Hongwei Guo for providing ein3 ebfl ebf2 mutant seeds. This work

306 was supported by National Institute of Health (GM047850) and National Key R\&D

307 Program of China (2017YFA0503800). The authors declare no conflict of interests.

308

309

310

311

312

313

314

315

316 
Literature Cited

Al-Sady B, Ni W, Kircher S, Schafer E, Quail PH (2006) Photoactivated phytochrome induces rapid PIF3 phosphorylation prior to proteasome-mediated degradation. Mol Cell 23: $439-446$

An F, Zhao Q, Ji Y, Li W, Jiang Z, Yu X, Zhang C, Han Y, He W, Liu Y, Zhang S, Ecker JR, Guo H (2010) Ethylene-induced stabilization of ETHYLENE INSENSITIVE3 and EIN3-LIKE1 is mediated by proteasomal degradation of EIN3 binding F-box 1 and 2 that requires EIN2 in Arabidopsis. Plant Cell 22: 2384-2401 Before It Gets Started: Regulating Translation at the 5' UTR. Comp Funct Genomics 2012: 475731

Bauer D, Viczian A, Kircher S, Nobis T, Nitschke R, Kunkel T, Panigrahi KC, Adam E,

Fejes E, Schafer E, Nagy F (2004) Constitutive photomorphogenesis 1 and multiple photoreceptors control degradation of phytochrome interacting factor 3, a transcription factor required for light signaling in Arabidopsis. Plant Cell 16: 1433-1445

Chen GH, Liu MJ, Xiong Y, Sheen J, Wu SH (2018) TOR and RPS6 transmit light signals to enhance protein translation in deetiolating Arabidopsis seedlings. Proc Natl Acad Sci U S A 115: 12823-12828

Christians MJ, Gingerich DJ, Hua Z, Lauer TD, Vierstra RD (2012) The light-response BTB1 and BTB2 proteins assemble nuclear ubiquitin ligases that modify phytochrome B and D signaling in Arabidopsis. Plant Physiol 160: 118-134

Dong J, Ni W, Yu R, Deng XW, Chen H, Wei N (2017) Light-Dependent Degradation of PIF3 by SCFEBF1/2 Promotes a Photomorphogenic Response in Arabidopsis. Curr Biol 27: $2420-2430$ e2426

348 Godoy Herz MA, Kubaczka MG, Brzyzek G, Servi L, Krzyszton M, Simpson C, 349 Brown J, Swiezewski S, Petrillo E, Kornblihtt AR (2019) Light Regulates Plant 350 Alternative Splicing through the Control of Transcriptional Elongation. Mol Cell 73: 
1066-1074 e1063

Hartmann L, Drewe-Boss P, Wiessner T, Wagner G, Geue S, Lee HC, Obermuller DM,

Kahles A, Behr J, Sinz FH, Ratsch G, Wachter A (2016) Alternative Splicing

354 Substantially Diversifies the Transcriptome during Early Photomorphogenesis and

355 Correlates with the Energy Availability in Arabidopsis. Plant Cell 28: 2715-2734

356 Kozak M (2001) Constraints on reinitiation of translation in mammals. Nucleic Acids Res

\section{$357 \quad$ 29: $5226-5232$}

358 Kurihara Y, Makita Y, Kawashima M, Fujita T, Iwasaki S, Matsui M (2018)

359 Transcripts from downstream alternative transcription start sites evade uORF-mediated

360 inhibition of gene expression in Arabidopsis. Proc Natl Acad Sci U S A 115: 7831-7836

361 Leivar P, Monte E (2014) PIFs: systems integrators in plant development. Plant Cell 26:

$362 \quad 56-78$

363 Leivar P, Monte E, Oka Y, Liu T, Carle C, Castillon A, Huq E, Quail PH (2008)

364 Multiple phytochrome-interacting bHLH transcription factors repress premature seedling

365 photomorphogenesis in darkness. Curr Biol 18: 1815-1823

366 Li J, Li G, Wang H, Wang Deng X (2011) Phytochrome signaling mechanisms.

367 Arabidopsis Book 9: e0148

368 Ni M, Tepperman JM, Quail PH (1998) PIF3, a phytochrome-interacting factor necessary

369 for normal photoinduced signal transduction, is a novel basic helix-loop-helix protein. Cell

370 95: $657-667$

371 Ni W, Xu SL, Tepperman JM, Stanley DJ, Maltby DA, Gross JD, Burlingame AL,

372 Wang ZY, Quail PH (2014) A mutually assured destruction mechanism attenuates light

373 signaling in Arabidopsis. Science 344: 1160-1164

374 Paik I, Yang S, Choi G (2012) Phytochrome regulates translation of mRNA in the cytosol.

375 Proc Natl Acad Sci U S A 109: 1335-1340

376 Park E, Kim Y, Choi G (2018) Phytochrome B Requires PIF Degradation and

377 Sequestration to Induce Light Responses Across a Wide Range of Light Conditions. Plant

378 Cell 30: 1277-1292 
379 Pham VN, Kathare PK, Huq E (2018) Phytochromes and Phytochrome Interacting

380 Factors. Plant Physiol 176: 1025-1038

381 Shi H, Shen X, Liu R, Xue C, Wei N, Deng XW, Zhong S (2016) The Red Light Receptor

382 Phytochrome B Directly Enhances Substrate-E3 Ligase Interactions to Attenuate Ethylene

383 Responses. Dev Cell 39: 597-610

384 Shikata H, Hanada K, Ushijima T, Nakashima M, Suzuki Y, Matsushita T (2014)

385 Phytochrome controls alternative splicing to mediate light responses in Arabidopsis. Proc

$386 \quad$ Natl Acad Sci U S A 111: 18781-18786

387 Shin J, Kim K, Kang H, Zulfugarov IS, Bae G, Lee CH, Lee D, Choi G (2009)

388 Phytochromes promote seedling light responses by inhibiting four negatively-acting

389 phytochrome-interacting factors. Proc Natl Acad Sci U S A 106: 7660-7665

390 Soy J, Leivar P, Gonzalez-Schain N, Sentandreu M, Prat S, Quail PH, Monte E (2012)

391 Phytochrome-imposed oscillations in PIF3 protein abundance regulate hypocotyl growth

392 under diurnal light/dark conditions in Arabidopsis. Plant J 71: 390-401

393 Soy J, Leivar P, Gonzalez-Schain N, Martin G, Diaz C, Sentandreu M, Al-Sady B,

394 Quail PH, Monte E (2016) Molecular convergence of clock and photosensory pathways 395 through PIF3-TOC1 interaction and co-occupancy of target promoters. Proc Natl Acad Sci

396 U S A 113: 4870-4875

397 Ushijima T, Hanada K, Gotoh E, Yamori W, Kodama Y, Tanaka H, Kusano M, 398 Fukushima A, Tokizawa M, Yamamoto YY, Tada Y, Suzuki Y, Matsushita T (2017)

399 Light Controls Protein Localization through Phytochrome-Mediated Alternative Promoter

$400 \quad$ Selection. Cell 171: 1316-1325 e1312

401 von Arnim AG, Jia Q, Vaughn JN (2014) Regulation of plant translation by upstream 402 open reading frames. Plant Sci 214: 1-12

403 Xin R, Zhu L, Salome PA, Mancini E, Marshall CM, Harmon FG, Yanovsky MJ,

404 Weigel D, Huq E (2017) SPF45-related splicing factor for phytochrome signaling promotes 405 photomorphogenesis by regulating pre-mRNA splicing in Arabidopsis. Proc Natl Acad Sci 406 U S A 114: E7018-E7027 
407 Yoo SD, Cho YH, Sheen J (2007) Arabidopsis mesophyll protoplasts: a versatile cell 408 system for transient gene expression analysis. Nat Protoc 2: 1565-1572

409 Zhang Y, Mayba O, Pfeiffer A, Shi H, Tepperman JM, Speed TP, Quail PH (2013) A 410 quartet of PIF bHLH factors provides a transcriptionally centered signaling hub that 411 regulates seedling morphogenesis through differential expression-patterning of shared target 412 genes in Arabidopsis. 
Figures and legends

A

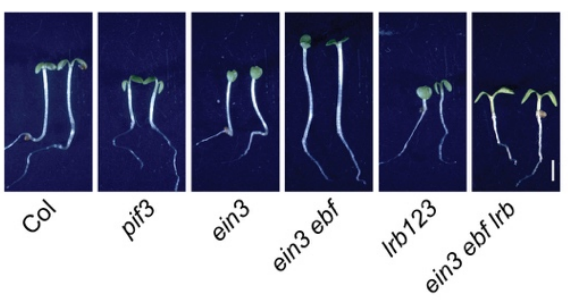

B

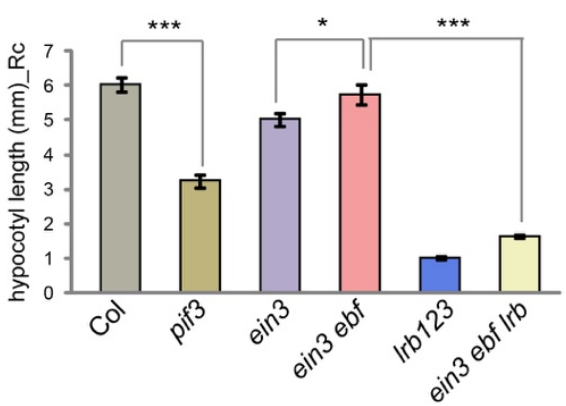

$\mathbf{F}$

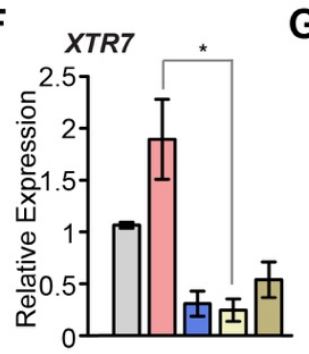

G $\quad$ xтH33

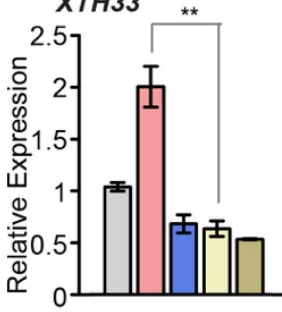

C

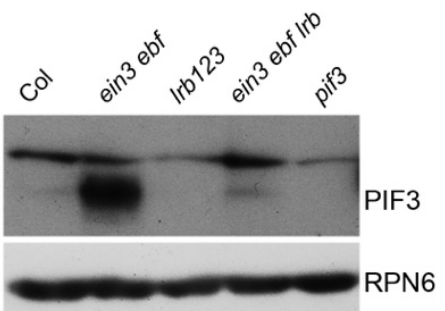

D

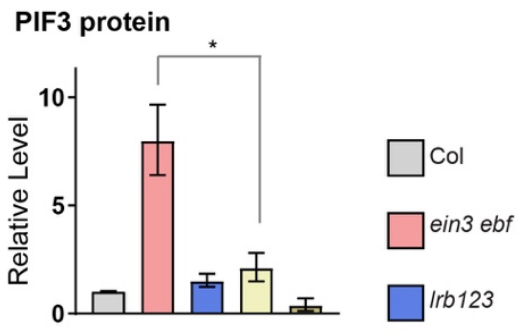

E

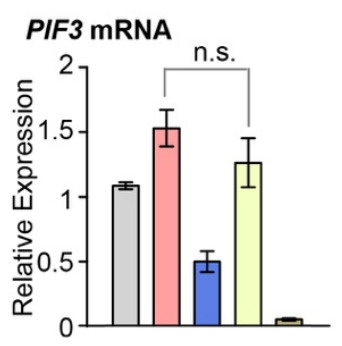

Figure 1. $L R B$ mutations resulted in a decrease of PIF3 protein and hypocotyl elongation in $\boldsymbol{e b f}$ mutant background under Rc. (A) Representative images of Col, pif3, ein3, ein3 ebf1 ebf2 (ein3 ebf), lrb1 lrb2 lrb3 (lrb123) and ein3 ebf1 ebf2 lrb1 lrb2 lrb3 (ein3 ebf lrb) seedlings grown under $10 \mu \mathrm{mol} / \mathrm{m}^{2} / \mathrm{s}$ continuous red light (Rc) for 4 days. Scale bar $=2 \mathrm{~mm}$. EIN3 is an ethylene response factor and a degradation target of EBF (Shi et al., 2016). Note: to offset the ethylene phenotype, the ebf mutant is represented by ein 3 ebf with ein 3 as its control. (B) Mean hypocotyl lengths of each genotype shown in (A). Data were shown in mean \pm SEM. (C and D) Accumulation of PIF3 protein in ein 3 ebf was suppressed in ein3 ebf lrb. Proteins were extracted from 4-day-old seedlings of indicated genotype grown under Rc, and western blots were performed using anti-PIF3 and anti-RPN6 antibodies. A representative western blot was shown in (C), and the mean relative PIF3 protein level in each genotype was shown in (D). Data were shown in mean \pm SEM of 3 biological replicates. Transcript levels of PIF3 (E), XTR7 (F), and XTH33 (G) in each genotype under Rc. Total RNAs were extracted from 4-day-old seedlings grown under Rc and reverse transcribed for RT-qPCR analyses. ACT2 was used as internal control. Data were shown in mean \pm SD of 3 biological replicates.

All statistic significances were calculated by Student's $t$ test: n.s., $\mathrm{p}>0.05 ; *, \mathrm{p}<0.05$; ***, $\mathrm{p}<0.001$. 
A

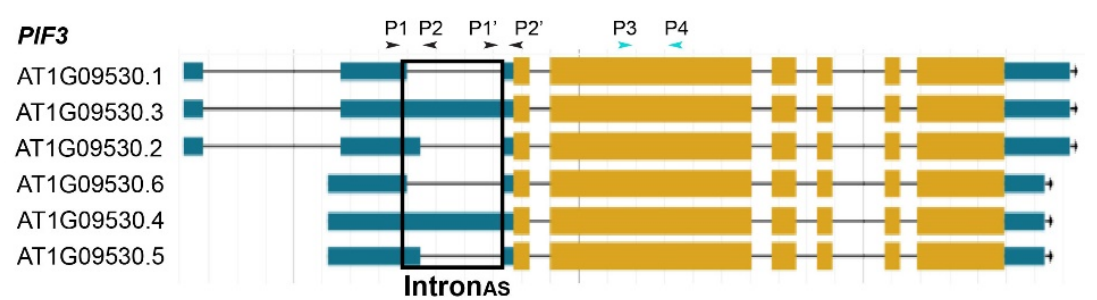

B

C

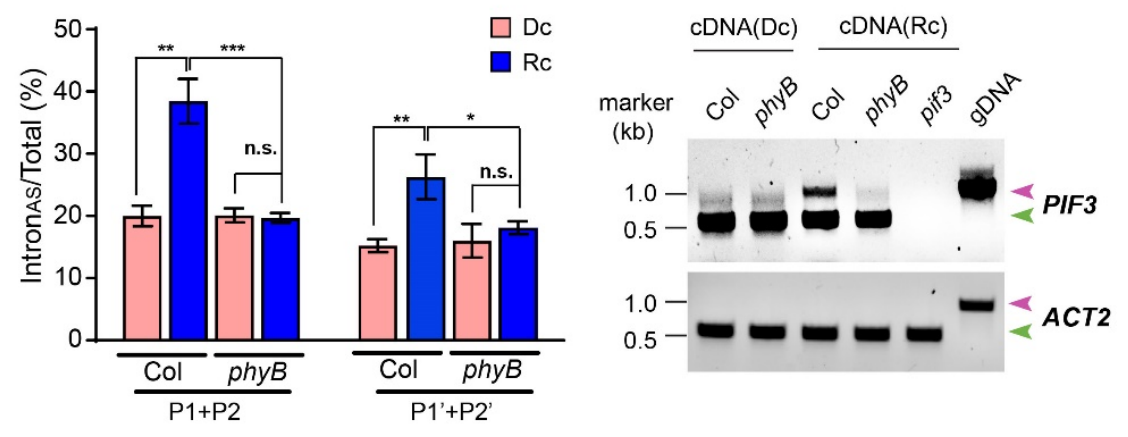

Figure 2. Light induces Intron $_{\mathrm{AS}}$ retention in PIF3 mRNA via phyB. (A) The schematic diagram of various PIF3 pre-mRNAs (Araport) and the primer pairs used for RT-qPCR. (B) The relative percentages of Intron $_{\mathrm{AS}}$ retained PIF3 mRNA (represented by $\mathrm{P} 1+\mathrm{P} 2$ or P1'+P2') to total PIF3 mRNA (represented by $\mathrm{P} 3+\mathrm{P} 4)$ in $\mathrm{Col}$ and $p h y B-9$ (phyB). cDNA was made from seedlings grown in the dark (Dc) or in $10 \mu \mathrm{mol} / \mathrm{m}^{2} / \mathrm{s}$ continuous red light (Rc) for 4 days. The RT-qPCR data are shown as the mean \pm SD of 3 biological replicates. Statistic significances were calculated by Student's $t$ test: n.s., $\mathrm{p}>0.05$; $^{*}, \mathrm{p}<0.05$; ** $\mathrm{p}<0.01$; ***, $<<0.001$. (C) The

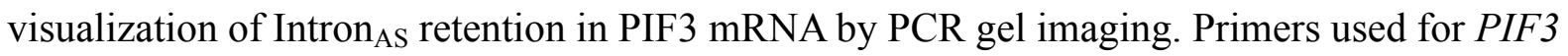
are P1 and P4 shown in (A). Primers used for ACT2 are ACT2I1gelf and ACT2I1gelr. Arrows in pink and green represent PCR products with and without Intron $_{\mathrm{AS}}$ retention, respectively. 
A

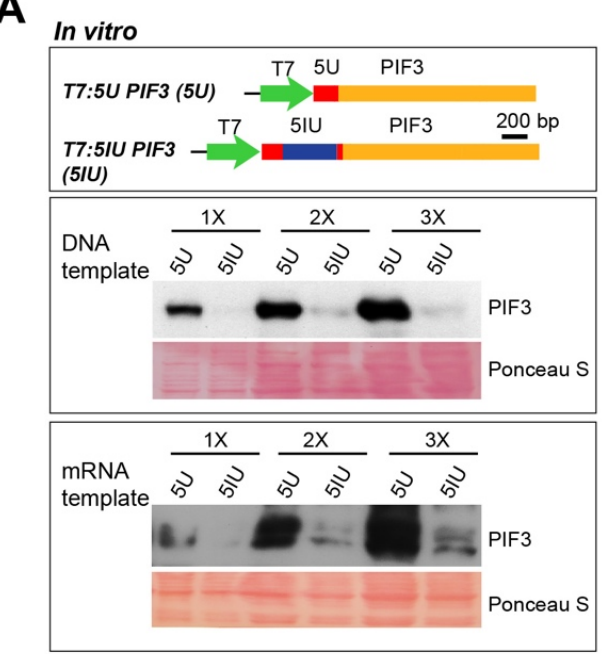

C

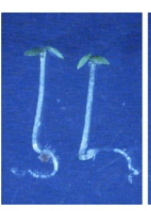

Col

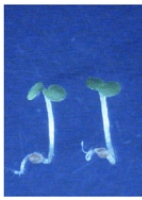

\#3

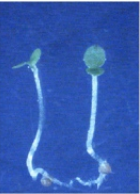

\#4

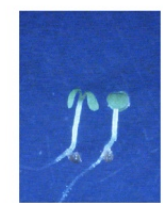

pif3-3

D

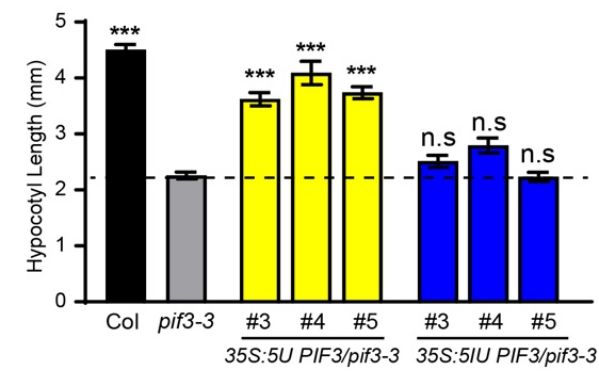

B

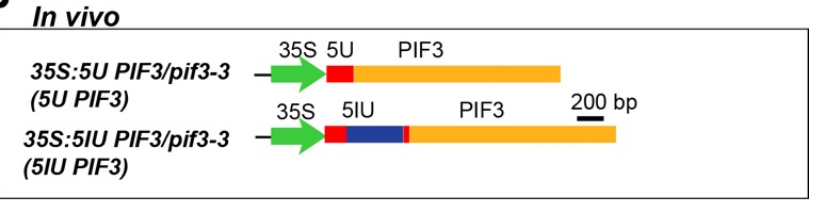

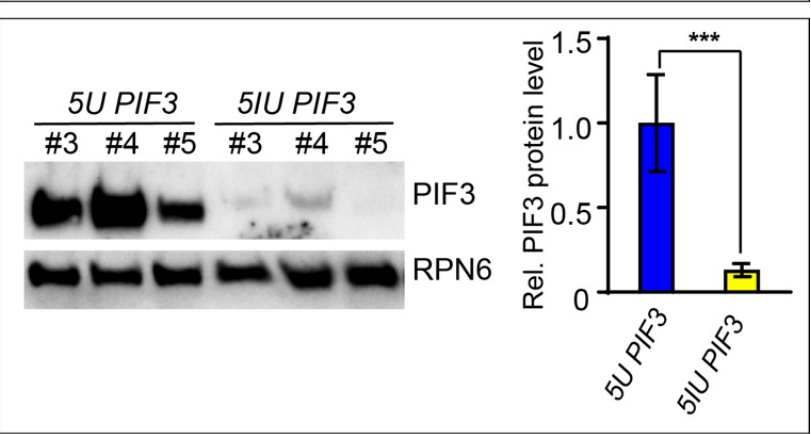

E
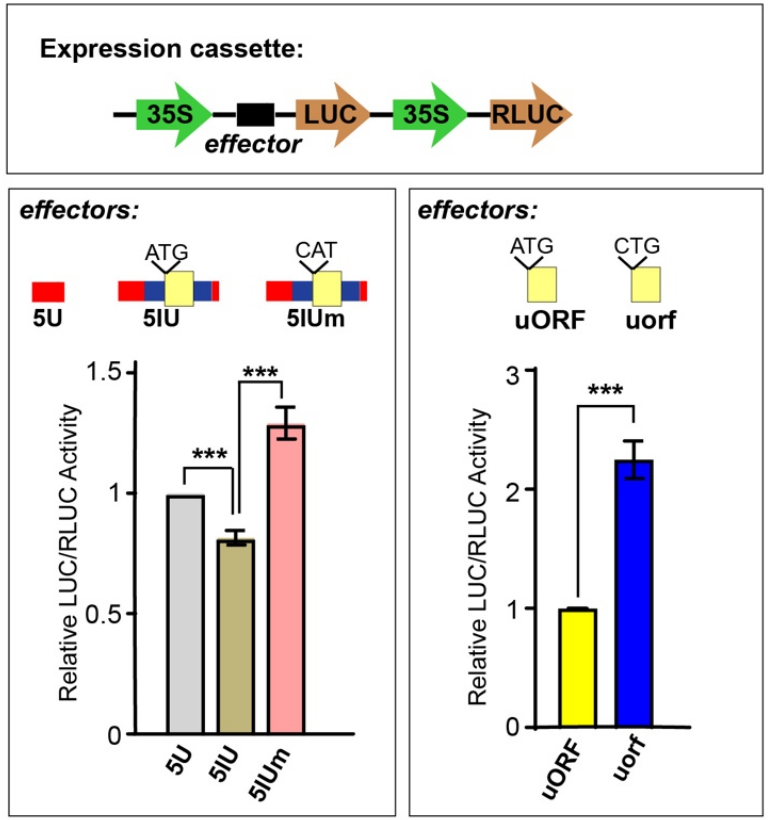

Figure 3. Intron $_{\mathrm{AS}}$ retention inhibits PIF3 mRNA translation via uORFs. (A) Intron $_{\mathrm{AS}}$ retention in PIF3 5'UTR inhibits protein translation in vitro. The schematic diagram shows the templates used for in vitro translation. DNA or in vitro transcribed mRNA was used as template for in vitro translation, and products were analyzed by anti-PIF3 western blots. $1 \times, 2 \times$ and $3 \times$ indicates the fold increasing amounts of in vitro translation samples that were loaded. (B) Intron $_{\mathrm{AS}}$ in the PIF3 5'UTR inhibits PIF3 protein production in vivo. The schematic diagram shows the expression cassettes used for generating transgenic plants in the pif3-3 background. Proteins extracted from 3-day dark grown seedlings were analyzed by anti-PIF3 western blots, with RPN6 as a loading control. Mean relative PIF3 protein levels were normalized to the corresponding RPN6 control and total PIF3 mRNA level. Data are shown as the mean \pm SD of 3 independent transgenic lines. The value in $5 U$ PIF3 transgenic plants was set as 1.0. (C and 
D) $5 U$ PIF3 transgene, but not 5IU PIF3, could rescue the phenotypes of pif3-3 under Rc. Three-day-old seedlings grown under Rc were photographed (C), and hypocotyl length was measured (D). Data were shown as the mean \pm SEM of at least 30 seedlings. Statistical significance was calculated against pif3-3 using Student's $t$ test: n.s., $\mathrm{p}>0.05$; ***, $\mathrm{p}<0.001$. (E) The constructs carrying indicated effectors were expressed in Arabidopsis protoplasts, and the ratio of LUC activity to RLUC activity was determined. Data were shown as the mean $\pm \mathrm{SD}$ of 4 biological replicates. All statistical significances were calculated by Student's $t$ test: ***, $\mathrm{p}<0.001$. 
A

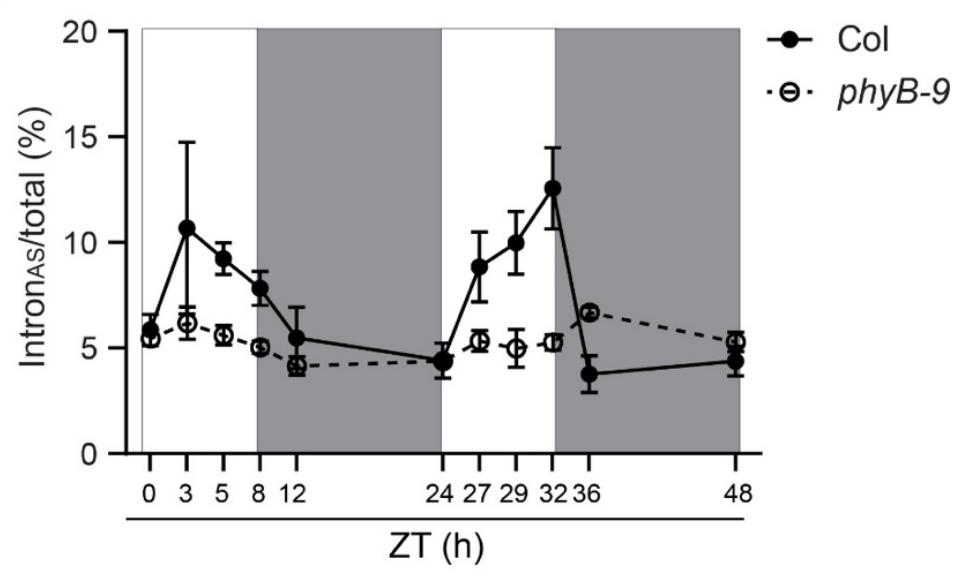

B

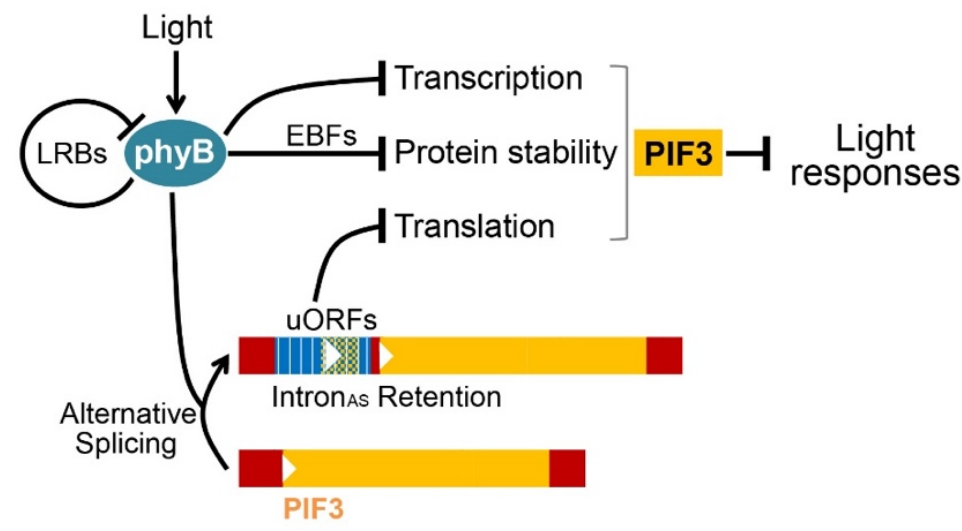

Figure 4. The phyB-dependent PIF3 AS-uORF plays a role in regulating PIF3 levels, in conjunction with other regulatory mechanisms (A) Diurnal regulation of Intron $_{\mathrm{AS}}$ retention in PIF3 mRNA. Col seedlings were grown under short day (8-hr light/16-hr dark) condition, and samples were harvested at indicated Zeitgeber Time (ZT) from 4 day old plants. Total RNA was extracted and reverse transcribed, and cDNA was used for qPCR analysis. Data were shown in mean $\pm \operatorname{sem}(\mathrm{n}=3$ biological replicates). (B) A summary diagram showing that light-activated phyB reduces PIF3 protein level in multiple ways to stimulate light responses. In addition to triggering transcriptional down-regulation and EBF-mediated protein degradation of PIF3, phyB can also induce alternative splicing in light-grown plants that results in the retention of Intron $_{\mathrm{AS}}$ (shown in blue) in the 5'UTR of PIF3 mRNA. The uORFs inside the Intron $_{\mathrm{AS}}$ sequence inhibit translation of PIF3 main ORF (shown in yellow). The 5' and 3' UTR region of PIF3 mRNA are indicated in red. LRB E3 ligases may work as a negative feedback system to control phyB protein levels and attenuate excessive light activation. 


\section{Parsed Citations}

A-Sady B, Ni W, Kircher S, Schafer E, Quail PH (2006) Photoactivated phytochrome induces rapid PIF3 phosphorylation prior to proteasome-mediated degradation. Mol Cell 23: 439-446

Pubmed: Author and Title

Google Scholar: Author Only Title Only Author and Title

An F, Zhao Q, Ji Y, Li W, Jiang Z, Yu X, Zhang C, Han Y, He W, Liu Y, Zhang S, Ecker JR, Guo H (2010) Ethylene-induced stabilization of ETHYLENE INSENSITIVE3 and EIN3-LIKE1 is mediated by proteasomal degradation of EIN3 binding F-box 1 and 2 that requires EIN2 in Arabidopsis. Plant Cell 22: 2384-2401

Pubmed: Author and Title

Google Scholar: Author Only Title Only Author and Title

Araujo PR, Yoon K, Ko D, Smith AD, Qiao M, Suresh U, Burns SC, Penalva LO (2012) Before It Gets Started: Regulating Translation at the 5' UTR. Comp Funct Genomics 2012: 475731

Pubmed: Author and Title

Google Scholar: Author Only Title Only Author and Title

Bauer D, Viczian A, Kircher S, Nobis T, Nitschke R, Kunkel T, Panigrahi KC, Adam E, Fejes E, Schafer E, Nagy F (2004) Constitutive photomorphogenesis 1 and multiple photoreceptors control degradation of phytochrome interacting factor 3 , a transcription factor required for light signaling in Arabidopsis. Plant Cell 16: 1433-1445

Pubmed: Author and Title

Google Scholar: Author Only Title Only Author and Title

Chen GH, Liu MJ, Xiong Y, Sheen J, Wu SH (2018) TOR and RPS6 transmit light signals to enhance protein translation in deetiolating Arabidopsis seedlings. Proc Natl Acad Sci U S A 115: 12823-12828

Pubmed: Author and Title

Google Scholar: Author Only Title Only Author and Title

Christians MJ, Gingerich DJ, Hua Z, Lauer TD, Vierstra RD (2012) The light-response BTB1 and BTB2 proteins assemble nuclear ubiquitin ligases that modify phytochrome B and D signaling in Arabidopsis. Plant Physiol 160: 118-134

Pubmed: Author and Title

Google Scholar: Author Only Title Only Author and Title

Dong J, Ni W, Yu R, Deng XW, Chen H, Wei N (2017) Light-Dependent Degradation of PIF3 by SCFEBF1/2 Promotes a

Photomorphogenic Response in Arabidopsis. Curr Biol 27: 2420-2430 e2426

Pubmed: Author and Title

Google Scholar: Author Only Title Only Author and Title

Godoy Herz MA, Kubaczka MG, Brzyzek G, Servi L, Krzyszton M, Simpson C, Brown J, Swiezewski S, Petrillo E, Kornblihtt AR (2019)

Light Regulates Plant Aternative Splicing through the Control of Transcriptional Elongation. Mol Cell 73: 1066-1074 e1063

Pubmed: Author and Title

Google Scholar: Author Only Title Only Author and Title

Hartmann L, Drewe-Boss P, Wiessner T, Wagner G, Geue S, Lee HC, Obermuller DM, Kahles A, Behr J, Sinz FH, Ratsch G, Wachter A (2016) Alternative Splicing Substantially Diversifies the Transcriptome during Early Photomorphogenesis and Correlates with the

Energy Availability in Arabidopsis. Plant Cell 28: 2715-2734

Pubmed: Author and Title

Google Scholar: Author Only Title Only Author and Title

Kozak M (2001) Constraints on reinitiation of translation in mammals. Nucleic Acids Res 29: 5226-5232

Pubmed: Author and Title

Google Scholar: Author Only Title Only Author and Title

Kurihara Y, Makita Y, Kawashima M, Fujita T, Iwasaki S, Matsui M (2018) Transcripts from downstream alternative transcription start sites evade uORF-mediated inhibition of gene expression in Arabidopsis. Proc Natl Acad Sci U S A115: 7831-7836

Pubmed: Author and Title

Google Scholar: Author Only Title Only Author and Title

Leivar P, Monte E (2014) PIFs: systems integrators in plant development. Plant Cell 26: 56-78

Pubmed: Author and Title

Google Scholar: Author Only Title Only Author and Title

Leivar P, Monte E, Oka Y, Liu T, Carle C, Castillon A, Huq E, Quail PH (2008) Multiple phytochrome-interacting bHLH transcription factors repress premature seedling photomorphogenesis in darkness. Curr Biol 18: 1815-1823

Pubmed: Author and Title

Google Scholar: Author Only Title Only Author and Title

Li J, Li G, Wang H, Wang Deng X (2011) Phytochrome signaling mechanisms. Arabidopsis Book 9: e0148

Pubmed: Author and Title

Google Scholar: Author Only Title Only Author and Title

Ni M, Tepperman JM, Quail PH (1998) PIF3, a phytochrome-interacting factor necessary for normal photoinduced signal transduction, is a novel basic helix-loop-helix protein. Cell 95: 657-667 
Ni W, Xu SL, Tepperman JM, Stanley DJ, Maltby DA, Gross JD, Burlingame AL, Wang ZY, Quail PH (2014) Amutually assured destruction mechanism attenuates light signaling in Arabidopsis. Science 344: 1160-1164

Pubmed: Author and Title

Google Scholar: Author Only Title Only Author and Title

Paik I, Yang S, Choi G (2012) Phytochrome regulates translation of mRNA in the cytosol. Proc Natl Acad Sci U S A109: 1335-1340

Pubmed: Author and Title

Google Scholar: Author Only Title Only Author and Title

Park E, Kim Y, Choi G (2018) Phytochrome B Requires PIF Degradation and Sequestration to Induce Light Responses Across a Wide Range of Light Conditions. Plant Cell 30: 1277-1292

Pubmed: Author and Title

Google Scholar: Author Only Title Only Author and Title

Pham VN, Kathare PK, Huq E (2018) Phytochromes and Phytochrome Interacting Factors. Plant Physiol 176: 1025-1038

Pubmed: Author and Title

Google Scholar: Author Only Title Only Author and Title

Shi H, Shen X, Liu R, Xue C, Wei N, Deng XW, Zhong S (2016) The Red Light Receptor Phytochrome B Directly Enhances Substrate-E3 Ligase Interactions to Attenuate Ethylene Responses. Dev Cell 39: 597-610

Pubmed: Author and Title

Google Scholar: Author Only Title Only Author and Title

Shikata H, Hanada K, Ushijima T, Nakashima M, Suzuki Y, Matsushita T (2014) Phytochrome controls alternative splicing to mediate light responses in Arabidopsis. Proc Natl Acad Sci U S A111: 18781-18786

Pubmed: Author and Title

Google Scholar: Author Only Title Only Author and Title

Shin J, Kim K, Kang H, Zulfugarov IS, Bae G, Lee CH, Lee D, Choi G (2009) Phytochromes promote seedling light responses by inhibiting four negatively-acting phytochrome-interacting factors. Proc Natl Acad Sci U S A 106: 7660-7665

Pubmed: Author and Title

Google Scholar: Author Only Title Only Author and Title

Soy J, Leivar P, Gonzalez-Schain N, Sentandreu M, Prat S, Quail PH, Monte E (2012) Phytochrome-imposed oscillations in PIF3 protein abundance regulate hypocotyl growth under diurnal light/dark conditions in Arabidopsis. Plant J 71: 390-401

Pubmed: Author and Title

Google Scholar: Author Only Title Only Author and Title

Soy J, Leivar P, Gonzalez-Schain N, Martin G, Diaz C, Sentandreu M, A-Sady B, Quail PH, Monte E (2016) Molecular convergence of clock and photosensory pathways through PIF3-TOC1 interaction and co-occupancy of target promoters. Proc Natl Acad Sci U S A113: 4870-4875

Pubmed: Author and Title

Google Scholar: Author Only Title Only Author and Title

Ushijima T, Hanada K, Gotoh E, Yamori W, Kodama Y, Tanaka H, Kusano M, Fukushima A, Tokizawa M, Yamamoto YY, Tada Y, Suzuki Y, Matsushita T (2017) Light Controls Protein Localization through Phytochrome-Mediated Alternative Promoter Selection. Cell 171: 13161325 e 1312

Pubmed: Author and Title

Google Scholar: Author Only Title Only Author and Title

von Arnim AG, Jia Q, Vaughn JN (2014) Regulation of plant translation by upstream open reading frames. Plant Sci 214: 1-12

Pubmed: Author and Title

Google Scholar: Author Only Title Only Author and Title

Xin R, Zhu L, Salome PA, Mancini E, Marshall CM, Harmon FG, Yanovsky MJ, Weigel D, Huq E (2017) SPF45-related splicing factor for phytochrome signaling promotes photomorphogenesis by regulating pre-mRNA splicing in Arabidopsis. Proc Natl Acad Sci U S A114: E7018-E7027

Pubmed: Author and Title

Google Scholar: Author Only Title Only Author and Title

Yoo SD, Cho YH, Sheen J (2007) Arabidopsis mesophyll protoplasts: a versatile cell system for transient gene expression analysis. Nat Protoc 2: 1565-1572

Pubmed: Author and Title

Google Scholar: Author Only Title Only Author and Title

Zhang Y, Mayba O, Pfeiffer A, Shi H, Tepperman JM, Speed TP, Quail PH (2013) Aquartet of PIF bHLH factors provides a transcriptionally centered signaling hub that regulates seedling morphogenesis through differential expression-patterning of shared target genes in Arabidopsis. 